\title{
Dynamic Characterization of a Radio Communication Tower with a FBG Based Accelerometer
}

\author{
Paulo Antunes ${ }^{1,2}$, Rui Travanca ${ }^{3}$, Humberto Varum ${ }^{3}$, Paulo André ${ }^{1,2}$ \\ ${ }^{1}$ Instituto de Telecomunicações, Campus de Santiago, 3810-193 Aveiro, Portugal: \\ ${ }^{2}$ Departamento de Física, Campus de Santiago, 3810-193 Aveiro, Portugal; \\ ${ }^{3}$ Departamento de Engenharia Civil, Campus de Santiago, 3810-193 Aveiro, Portugal; \\ pandre@av.it.pt
}

\begin{abstract}
The dynamic monitoring of a tower for mobile communications with 50 meters high using a bi-axial optical accelerometer is reported. The resonant frequencies for the first modes were obtained, being consistent with the expected values.
\end{abstract}

\section{Introduction}

Towers are one of the most important physical supports for the installation of radio equipment used for the emission of electromagnetic waves for services such as radio, radar, television and mobile communications. In the last decades, the demand for this type of structures was determined particularly by the advent of radio, radar and television. More recently, this growth has become exponential due to the towers demand for using in mobile communication networks. Unfortunately, the number of anomalies observed in such structures is high if compared with other structures of similar social and economic importance [1-4].

Particularly, for economic and functional reasons of their own nature, towers are lightweight structures with high slenderness and great flexibility. The predominant loads are natural loads as wind and/or ice. Wind is a dynamic action and slender structures are sensitive to turbulent fluctuations in the flow, causing both background and resonant response. The resonant response becomes important when these structures have a natural frequency below $1 \mathrm{~Hz}$. Therefore, dynamic analysis is needed to determine the resonance response that could be significant compared with the background response. Besides the in-wind dynamic response caused by the turbulence of the wind, there are other dynamic mechanisms that need to be considered. The most common of these occurs on towers of cylindrical cross-section and is caused by the regular shedding of vortices. When the frequency of vortex shedding coincides with the natural frequency of the structure, sustained vibrations can occur. Although know for a long time, the crosswind forcing mechanism are complex and the procedures available to calculate the structure response are still rather crude and empirical $[2,4,5]$.

Optical sensors are an excellent choice for the dynamic monitoring of high slender structures, a vital procedure to assess their behavior, control its performance and certainly to a better understanding of their response. Fiber Bragg Grating (FBG) based sensors present intrinsic advantages which makes them ideal for multiplexed networks of different types of sensors (humidity, strain, pressure, velocity, acceleration, temperature, etc.) without the need of heavy cabling [6,7]. Also, FBG sensors are immune to the antennas electromagnetic radiation and do not interfere with their proper work. Besides, FBG take advantage of being an all-in-fiber technology, presenting very low attenuation and, consequently, being suitable to be used in remote monitoring.

In this work we use an optical FBG based biaxial accelerometer to obtain the frequencies for the two perpendicular directions parallel to the ground. These values can be used to calibrate numerical finite element models to represent the structural behavior of this type of structures, that can be used to identify eventual structural damages or deficiencies, to develop parametric vulnerability studies, to develop and optimize new structural solutions and, certainly, to a better understanding of the in-wind and cross-wind response for this type of structures.

\section{Monitored tower and setup}

In this test, it was chosen a steel monopole with 50 meters high, showed in figure 1a, composed by five modules with tubular cross-section, linked with bolted connections. The steel monopole is fixed at its base to a reinforced concrete foundation block with dimensions $4.00 \times 4.00 \mathrm{~m}$ in plan and $2.25 \mathrm{~m}$ in depth.

The optical FBG based bi-axial accelerometer was fixed on a rigid steel support system and mounted at the top of the tower with a bolted connection. All testing works were developed in less than 3 hours, including climbing, assembling, setup installation, measuring, disassembling and descend of men and equipment. The monitoring setup is illustrated in figure $1 \mathrm{~b}$ and consists in an optical broadband source ALS-CL-17-B-FA from Amonics, an optical circulator and a spectrometer from Ibsen, model I-MON E, acquiring at a sample rate of $950 \mathrm{~Hz}$. The measurements took about 95 seconds during which small mechanical horizontal impulses were applied on the top of the tower, 
approximately 2 meters below its top. Using a bi-axial optical accelerometer it was possible to measure acceleration time histories in two independent horizontal directions.

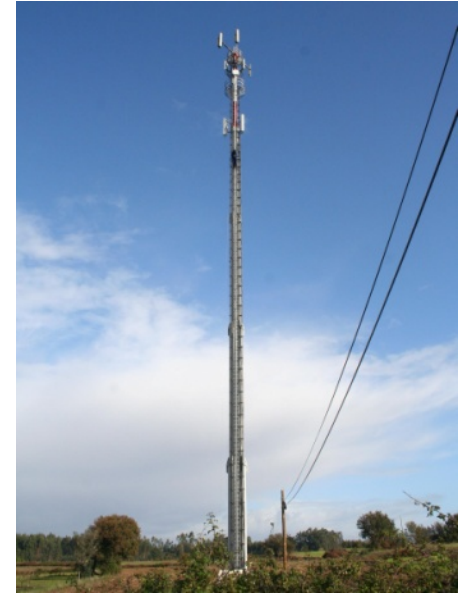

a)

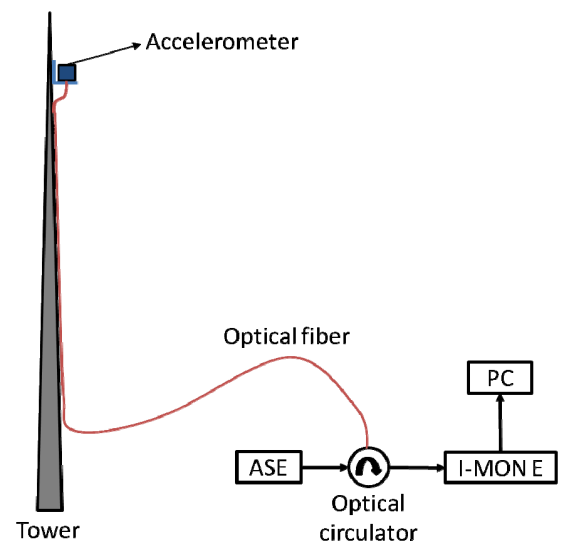

b)

Fig. 1. Monitored steel monopole: a) general view, b) Monitoring setup.

\section{Results}

In figure 2 is presented the acceleration data over time acquired during the application of small mechanical horizontal impulses. The acceleration was measured for the two sensitive directions of the accelerometer.

The natural frequencies of the structure can be obtained identifying the peaks on the frequencies spectra, which are obtained from fast Fourier transform (FFT) of the acceleration time histories recorded. The frequencies spectra obtained are shown in figure 3 for both sensitive directions.

From the data presented in figure 3 it is possible to indentify the values of the first three frequencies of the structure for each direction. Although, the first natural frequency is the most important since is the most representative of the structure dynamic response, the values for the following modes can also assist in the calibration of the numerical model, since these structures are not perfectly symmetric in terms of stiffness and mass distribution. The results obtained for each direction are consistent, and are in the range of the values expected for this type of structures.

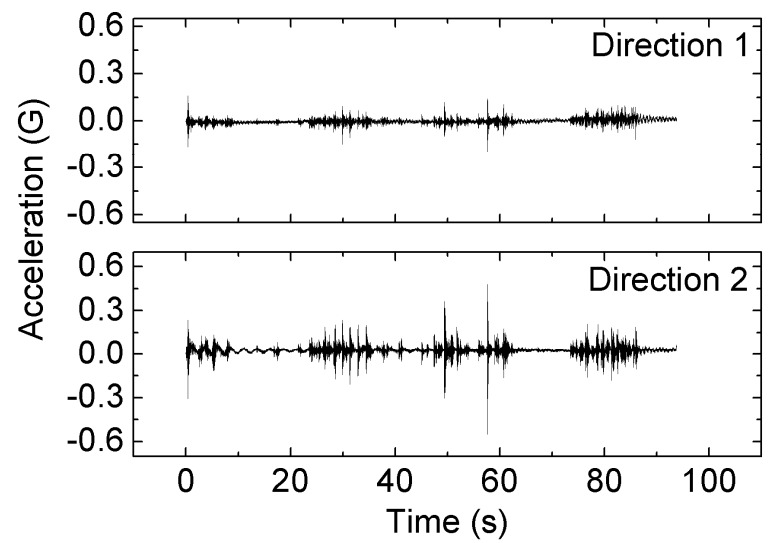

Fig. 2. Measured acceleration time histories in both horizontal directions. 


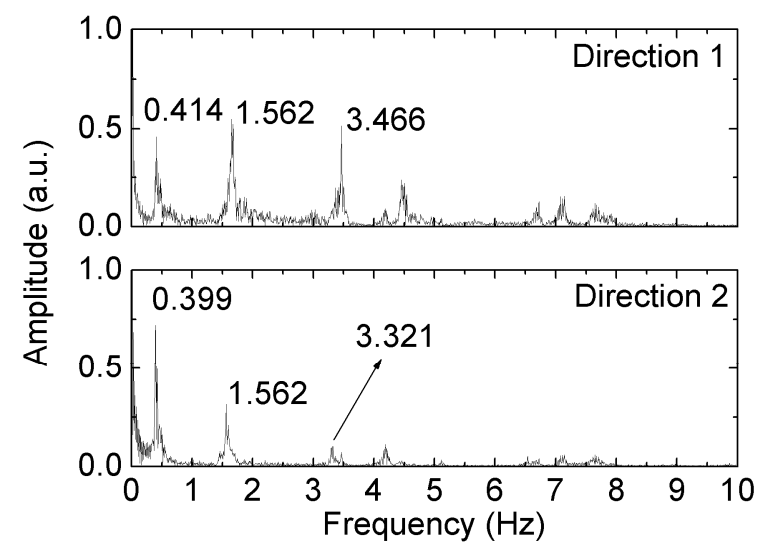

Fig. 3. Frequencies spectra for acceleration time histories recorded in both directions.

\section{Conclusions}

In this work, the use of optical technology for the dynamic characterization of a steel tower has been demonstrated. The FBG based accelerometer was able to measure acceleration in two independent horizontal directions, from which it was possible to obtain the natural frequencies of the structure. The values found for those natural frequencies are in the range of frequencies expected for this type of structures. This data can be used to calibrate numerical finite element models that represent the structural behavior of this type of towers. Accurate and calibrate numerical models can be used to identify eventual structural damages or deficiencies, to develop parametric vulnerability studies, to develop and optimize new structural solutions, and certainly to reach a better understanding of the in-wind and cross-wind response of this type of structures.

\section{Acknowledgments}

P. F. C. Antunes acknowledge the financial support from Fundação para a Ciência e Tecnologia (FCT) through the $\mathrm{PhD}$ fellowship SFRH/BD/41077/2007. The support of Vodafone Portugal and Telcabo is most gratefully acknowledged.

\section{References}

[1] B. Smith, "50 years in the design of towers and masts. From IASS recommendations to current procedures", in proceedings of the International Association for Shell and Spatial Structures (IASS) Symposium, Valencia, 28 September - 2 October (2009).

[2] B. Smith, Communication Structures (Thomas Telford, London 2007).

[3] U. Støttrup-Andersen, "Masts and towers", in proceedings of the International Association for Shell and Spatial Structures (IASS) Symposium, Valencia, 28 September - 2 October (2009).

[4] R. Travanca "Radio communication towers. Pathologies and design", Master Thesis (University of Aveiro 2010) (in Portuguese).

[5] A. Davenport, "What makes a structure wind sensitive?", Wind Effects on Buildings and Structures, (Riera \& Davenport 1998).

[6] P. F. C. Antunes, et al., "Optical Fiber Accelerometer System for Structural Dynamic Monitoring," Sensors Journal, IEEE 9, 13471354 (2009).

[7] A. Othonos and K. Kalli, Fiber Bragg Gratings: Fundamentals and Applications in Telecommunications and Sensing (Artech House 1999). 